A

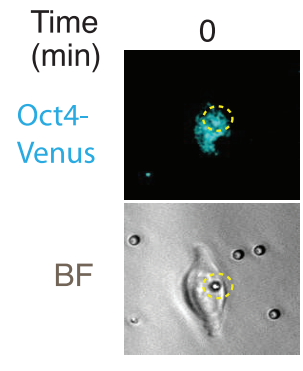

$\mathbf{B}$

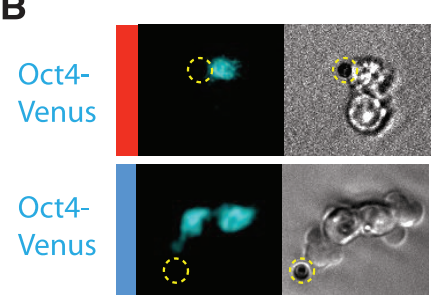

40
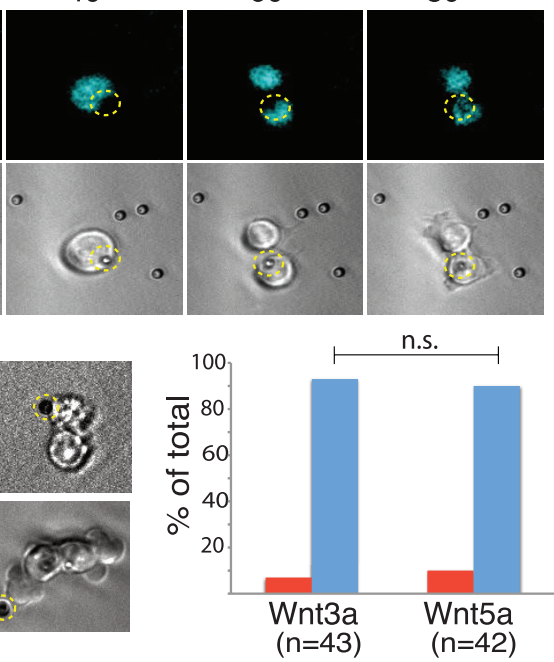

Fig. 4. Distal cells express markers of epiblast stem cell fate. (A) Selected frames from time-lapse imaging of a dividing Oct4-Venus reporter ES cell cocultured with a Wnt3a bead (indicated by a dashed yellow circle). (B) Divisions of Oct4-Venus ES cells cocultured with Wnt3a or Wnt5a beads were classified based on the relative expression of Venus, and plotted. Red bar: higher Venus abundance in the bead-proximal cell; blue bar: similar abundance of Venus in

C

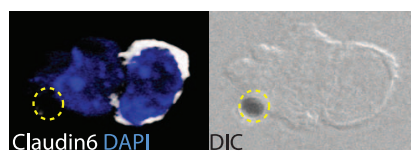

D
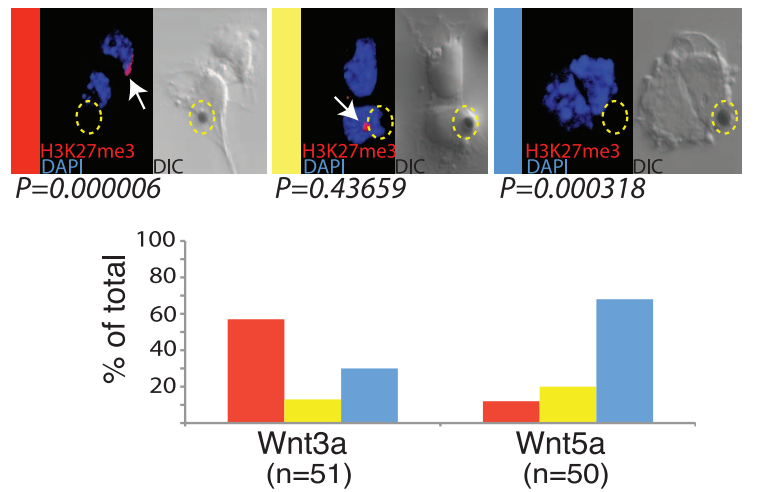

both cells. (C) Antibody staining for Claudin6 in an ES cell cocultured with a Wnt3a bead. (D) Representative images of antibody staining for H3K27me3 (arrows) in LF2 female ES cells cocultured with Wnt3a or Wnt5a beads. Dividing cells were classified based on location of the H3K27me3 stain, and plotted. Red bar: H3K27me3 stain in the bead-proximal cell; yellow bar: H3K27me3 stain in the bead-distal cell; blue bar: no H3K27me3 stain in any cell.
References and Notes

1. R. A. Neumüller, J. A. Knoblich, Genes Dev. 23, 2675 (2009).

2. A. D. Werts, B. Goldstein, Semin. Cell Dev. Biol. 22, 842 (2011).

3. T. Walston et al., Dev. Cell 7, 831 (2004).

4. B. Goldstein, H. Takeshita, K. Mizumoto, H. Sawa, Dev. Cell 10, 391 (2006).

5. K. Sugioka, K. Mizumoto, H. Sawa, Cell 146, 942 (2011).

6. D. ten Berge et al., Nat. Cell Biol. 13, 1070 (2011).

7. N. Sato, L. Meijer, L. Skaltsounis, P. Greengard, A. H. Brivanlou, Nat. Med. 10, 55 (2004).

8. Q.-L. Ying et al., Nature 453, 519 (2008).

9. A. Surani, J. Tischler, Nature 487, 43 (2012).

10. A. J. Mikels, R. Nusse, PLoS Biol. 4, e115 (2006).

11. S. Bahmanyar et al., Genes Dev. 22, 91 (2008).

12. Y. M. Yamashita, D. L. Jones, M. T. Fuller, Science 301 , 1547 (2003)

13. M. M. Mogensen, A. Malik, M. Piel, V. Bouckson-Castaing, M. Bornens, J. Cell Sci. 113, 3013 (2000).

14. X. Wang et al., Nature 461, 947 (2009).

15. M. Bornens, Science 335, 422 (2012).

16. Y. M. Yamashita, M. T. Fuller, J. Cell Biol. 180, 261 (2008).

17. T. A. Planchon et al., Nat. Methods 8, 417 (2011).

18. B. Payer et al., Genesis 44, 75 (2006).

19. Y. Toyooka, D. Shimosato, K. Murakami, K. Takahashi, H. Niwa, Development 135, 909 (2008).

20. K. Hayashi, S. M. Lopes, F. Tang, M. A. Surani, Cell Stem Cell 3, 391 (2008).

21. K. Arnold et al., Cell Stem Cell 9, 317 (2011).

22. A. M. Singh, T. Hamazaki, K. E. Hankowski, N. Terada, Stem Cells 25, 2534 (2007).

23. I. Chambers et al., Nature 450, 1230 (2007).

24. K. A. Kim et al., Science 309, 1256 (2005).

25. P. J. Tesar et al., Nature 448, 196 (2007).

26. G. Guo et al., Development 136, 1063 (2009)

27. K. Plath et al., Science 300, 131 (2003).

28. M. A. Hilliard, C. I. Bargmann, Dev. Cell 10, 379 (2006).

29. C. Korkut et al., Cell 139, 393 (2009).

Acknowledgments: These studies were supported by the Howard Hughes Medical Institute and by grants RC1-00133-1, RB4-05825, and TR1-02149 from the California Institute for Regenerative Medicine to R.N.; by NIH grant GM063702 to T.M.; and by the Center for Regenerative Therapies Dresden and grant DFG-SPP1356 to K.A. We thank the Stanford
Neuroscience Microscopy Service, supported by NIH NS069375. Patent applications are pending for the Bessel beam plane illumination microscopy (by E.B.) and for the Wnt, DKK, and $\mathrm{R}$ spondin1 immobilization technology and their applications (by S.].H. and R.N.). We also thank M. Drukker, T. Schroeder, and T. Stearns for discussions. We appreciate discussions and comments on the manuscript by C. Logan and J. Nelson. S.J.H. was supported by a fellowship from the Deutsche Forschungsgemeinschaft (DFG) and a Siebel scholarship.
Supplementary Materials

www.sciencemag.org/cgi/content/full/339/6126/1445/DC1 Materials and Methods

Figs. S1 to S12

Movies S1 to S10

References (30-33)

3 October 2012; accepted 12 February 2013

10.1126/science. 1231077

\title{
Type I Interferon Suppresses Type II Interferon-Triggered Human Anti-Mycobacterial Responses
}

Rosane M. B. Teles, ${ }^{1}$ Thomas G. Graeber, ${ }^{4}$ Stephan R. Krutzik, ${ }^{1}$ Dennis Montoya, ${ }^{1}$ Mirjam Schenk, ${ }^{1}$ Delphine J. Lee, ${ }^{5}$ Evangelia Komisopoulou, ${ }^{4}$ Kindra Kelly-Scumpia, ${ }^{1}$ Rene Chun, ${ }^{3}$ Shankar S. Iyer, ${ }^{2}$ Euzenir N. Sarno, ${ }^{6}$ Thomas H. Rea, ${ }^{7}$ Martin Hewison, ${ }^{3}$ John S. Adams, ${ }^{3}$ Stephen J. Popper, ${ }^{8}$ David A. Relman, ${ }^{8,9}$ Steffen Stenger, ${ }^{10}$ Barry R. Bloom, ${ }^{11}$ Genhong Cheng, ${ }^{2}$ Robert L. Modlin ${ }^{1,2 *}$

Type I interferons (IFN- $\alpha$ and IFN- $\beta$ ) are important for protection against many viral infections, whereas type II interferon (IFN- $\gamma$ ) is essential for host defense against some bacterial and parasitic pathogens. Study of IFN responses in human leprosy revealed an inverse correlation between IFN- $\beta$ and IFN- $\gamma$ gene expression programs. IFN- $\gamma$ and its downstream vitamin D-dependent antimicrobial genes were preferentially expressed in self-healing tuberculoid lesions and mediated antimicrobial activity against the pathogen Mycobacterium leprae in vitro. In contrast, IFN- $\beta$ and its downstream genes, including interleukin-10 (IL-10), were induced in monocytes by $M$. leprae in vitro and preferentially expressed in disseminated and progressive lepromatous lesions. The IFN- $\gamma$-induced macrophage vitamin D-dependent antimicrobial peptide response was inhibited by IFN- $\beta$ and by IL-10, suggesting that the differential production of IFNs contributes to protection versus pathogenesis in some human bacterial infections.

he identification of mechanisms of host resistance versus susceptibility is central to our ability to develop new approaches to prevent and/or treat human infectious diseases. In most instances, the human immune response restricts the infection, preventing or limiting the 
extent of disease. However, in some individuals the infection is not contained and instead progresses. Protective immunity against many intracellular bacteria depends on type 1 helper $\left(\mathrm{T}_{\mathrm{H}} 1\right)$ $\mathrm{T}$ cell responses, in particular the production of the type II interferon (IFN), IFN- $\gamma$, which can activate antimicrobial responses (l). However, the induction of type I IFNs, important for host defense against viral infections, is ineffective in the context of bacterial pathogens (2), such as

${ }^{1}$ Division of Dermatology, David Geffen School of Medicine at University of California, Los Angeles, CA 90095, USA. ²Department of Microbiology, Immunology and Molecular Genetics, David Geffen School of Medicine at University of California, Los Angeles, CA 90095, USA. ${ }^{3}$ UCLA/Orthopedic Hospital Department of Orthopedic Surgery, David Geffen School of Medicine at University of California, Los Angeles, CA 90095, USA. ${ }^{4}$ Crump Institute for Molecular Imaging, Institute for Molecular Medicine, Johnson Comprehensive Cancer Center, California NanoSystems Institute, Department of Molecular and Medical Pharmacology, University of California, Los Angeles, CA 90095, USA. ${ }^{5}$ Department of Translational Immunology, John Wayne Cancer Institute, Santa Monica, CA, USA. ${ }^{6}$ Department of Mycobacteriosis, Oswaldo Cruz Foundation, Rio de Janeiro, R], Brazil. ${ }^{7}$ Department of Dermatology, University of Southern California School of Medicine, Los Angeles, CA 90033, USA ${ }^{8}$ Department of Microbiology and Immunology and Department of Medicine, Stanford University School of Medicine, Stanford, CA 94305, USA. 'Veterans Affairs Palo Alto Health Care System, Palo Alto, CA 94304, USA. ${ }^{10}$ Institute for Medical Microbiology and Hygiene, University Hospital of Ulm, Germany. ${ }^{11}$ Harvard School of Public Health, Boston, MA 02115, USA.

*Corresponding author. E-mail: rmodlin@mednet.ucla.edu
Mycobacterium tuberculosis, and is associated with a greater extent of disease (3). These clinicalimmunological correlations suggest that type I IFNs can suppress IFN- $\boldsymbol{\gamma}$-induced antimicrobial responses in humans.

To explore the mechanisms by which type I IFNs may suppress IFN- $\gamma$-induced host defense responses, we chose leprosy, a disease of skin and nerves caused by the intracellular pathogen Mycobacterium leprae, as a model for understanding the dynamics of immune responses in skin lesions. The clinical presentations of leprosy compose a spectrum that correlates with the type of immune response induced. In the selfhealing tuberculoid (T-lep) form, the host immune response is able to effectively combat the pathogen, there are few skin lesions, and bacteria are rare. In the disseminated lepromatous (L-lep) form, the host immune response fails, resulting in numerous skin lesions characterized by abundant intracellular bacilli. T-lep lesions express $\mathrm{T}_{\mathrm{H}} 1$ cytokines including IFN- $\gamma$, whereas L-lep lesions are characterized by $\mathrm{T}_{\mathrm{H}} 2$ cytokines as well as interleukin-10 (IL-10) (4). Reversal reactions (RRs) represent a shift from the L-lep toward the T-lep form, accompanied by a reduction of bacilli in lesions and enhanced $\mathrm{T}_{\mathrm{H}} 1$ cytokine responses (5).

The gene expression profiles of skin lesions from leprosy lesions were first evaluated by principal component analysis and hierarchical clus- tering analysis (Fig. 1, A and B, and fig. S1), both revealing a distinct gene expression profile in L-lep lesions as compared with T-lep and RR lesions. Although the signal for all type I IFN mRNAs, including all 13 IFN- $\alpha$ genes and one IFN- $\beta$ gene, was within the microarray background noise resulting in absent calls, integration of the leprosy gene expression profiles with IFN-induced transcriptional profiles in healthy human peripheral blood mononuclear cells (PBMCs) (6) revealed that genes specifically induced by IFN- $\beta$, including IL-10, were significantly enriched in the L-lep gene expression profile. In contrast, IFN- $\gamma$ specific genes were significantly enriched in the T-lep (Fig. 1C and fig. S2) and RR lesions (fig. S3). The presence of a type I IFN gene expression signature in L-lep lesions was confirmed by analysis using the "interferome" database of IFN-regulated genes (7) (fig. S4).

An overall summation score of the IFN- $\beta$ and IFN- $\gamma$ profiles of each leprosy patient, calculated by using a gene voting approach $(8)$, revealed a significant inverse correlation between the IFNinducible programs; the IFN- $\beta$ profile greatest and the IFN- $\gamma$ profile lowest in L-lep lesions; and the IFN- $\gamma$ profile greatest and IFN- $\beta$ lowest in T-lep and RR lesions ( $r=-0.89$, Fig. 1D). Further analysis revealed in L-lep lesions an IFN$\beta \rightarrow \mathbb{I L}-10$ pathway (9), previously shown to inhibit IFN- $\gamma$ activation of macrophages in a mouse model
A
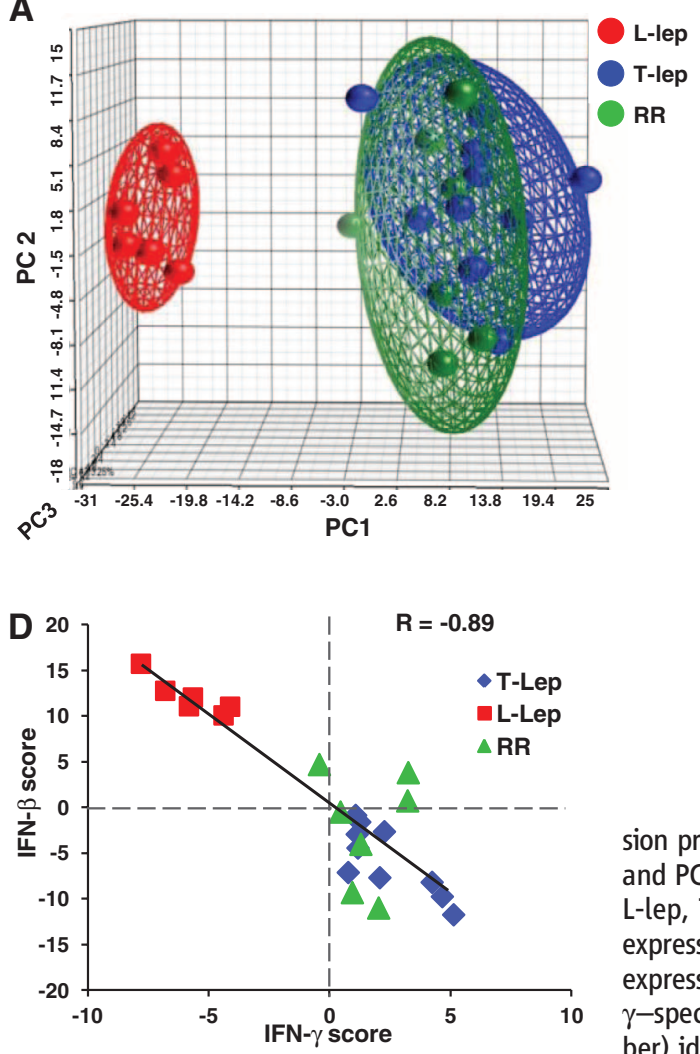

B

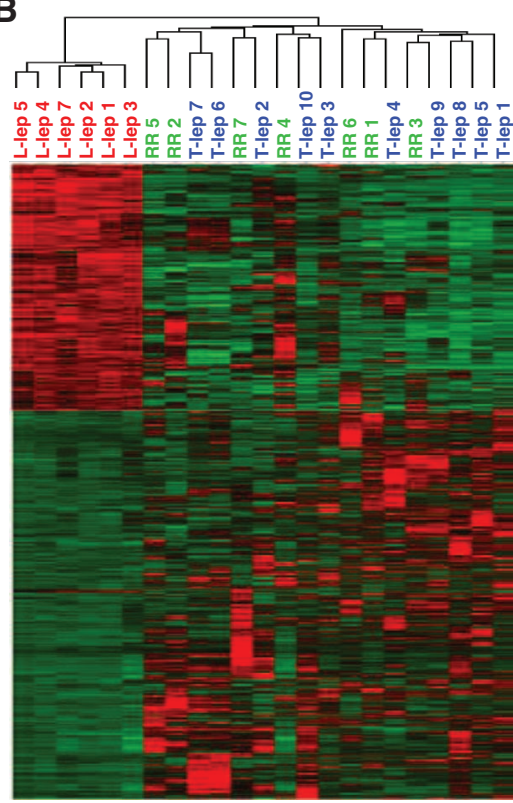

C
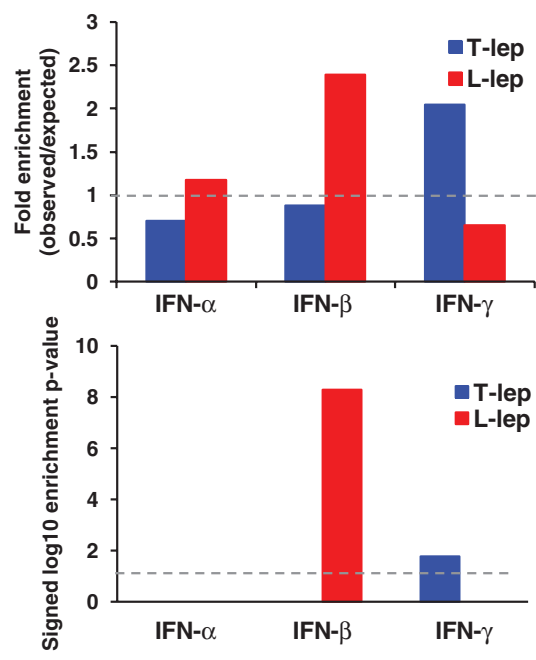

Fig. 1. IFN signatures in leprosy skin lesions. (A) Unsupervised principal component analysis (PCA) of L-lep, T-lep, and RR skin lesion gene expression profiles. Total three-dimensional PCA mapping as $81.5 \%$ of variance $(\mathrm{PC} 1=69.5 \%$, $\mathrm{PC2}=8.75 \%$, and $\mathrm{PC} 3=3.25 \%$ ). (B) Unsupervised clustering analysis using differentially expressed genes between L-lep, T-lep, and RR (coefficient of variation $\geq 1.0$ ). Individual squares represent the relative gene expression intensity of the given gene (rows) in a patient (columns), with red indicating an increase in expression and green a decrease. (C) Enrichment analysis of overlap between IFN- $\alpha-$, IFN- $\beta-$, and IFN$\gamma$-specific genes (induced and repressed; limited to genes modulated by only one IFN family member) identified in healthy human PBMCs and L-lep- and T-lep-specific leprosy lesion transcripts (fold change $\geq 1.5$ and $P \leq 0.05$ ). Dotted lines indicate either the expected fold enrichment of one (top) or the hypergeometric enrichment $P$ value of 0.05 (log $P=1.3$, bottom). Hypergeometric analyses were performed to determine fold enrichment (observed/expected) and signed log enrichment $P$ value (negative for deenriched). The Bonferroni multiple hypothesis test correction was applied for each group $(n=6)$. (D) IFN- $\beta-$ and IFN- $\gamma$-specific gene voting summation scores were calculated for individual patient lesions in the leprosy subtypes of L-lep, T-lep, and RR. 
of infection (10). In contrast, an IFN- $\gamma$-inducible vitamin D-dependent antimicrobial pathway (11) was found in the T-lep and RR lesions (fig. S5).

Analogous to leprosy, most individuals infected with $M$. tuberculosis localize and cure or contain the infection (latent tuberculosis); but in about $10 \%$ of patients the infection progresses to clinical disease, including pulmonary tuberculosis. A key study of gene expression profiles in peripheral blood from tuberculosis patients found a differential expression of an IFN-inducible gene profile in patients with active versus latent disease (3); the training cohort from this study is referred to here as TB1. Integration of the TB1 gene expression profiles with the PBMC-defined IFN-specific genes revealed an IFN- $\beta$-induced gene program in active tuberculosis (figs. S6 and S7). Comparison of the IFN- $\beta$ signature in L-lep lesions versus TB1 as well as a second independent active tuberculosis cohort, TB2 (12), identified a common signature of 16 IFN- $\beta$-induced genes (hypergeometric $P$ value $=0.0007$, fig. S7). Further integration with the gene expression profile of IL-10-treated monocytes revealed that 9 of 16 IFN- $\beta$-induced genes in the leprosy and tuberculosis common signature are also downstream of IL-10 (hypergeometric $P$ value $=0.02$, fig. S7). The data indicate that a common IFN- $\beta$ inducible gene program correlates with extent of disease in both leprosy and tuberculosis, suggesting that IFN- $\beta$ is a common factor contributing to pathogenesis in the two distinct mycobacterial diseases.

Fig. 2. IFN- $\beta$ is up-regulated in L-lep lesions. (A) Total mRNA was isolated from L-lep $(n=10)$, T-lep $(n=10)$, and RR $(n=10)$ skin lesions; and the IFN- $\beta$, IFNAR1, and IFN- $\gamma$ mRNA levels were analyzed by quantitative PCR (qPCR). The levels of IFN- $\beta$, IFNAR1 and IFN- $\gamma$ were normalized to glyceraldehyde-3-phosphate dehydrogenase (GAPDH) levels in the same tissue. Statistical significance was calculated by one-way ANOVA followed by the posttest, Newman-Keuls multiple comparison test for IFN- $\beta$ and IFNR1, and KruskalWallis followed by Dunn's multiple comparison test for IFN- $\gamma$. ${ }^{* *} P \leq 0.01$; ${ }^{*} P \leq 0.05$. (B) IFN- $\beta$ and IFNAR1 expression were detected in leprosy lesions (T-lep, L-lep, and RR); one representative labeled section is shown out of at least five; scale bars, $40 \mu \mathrm{m}$. Original magnification, 100x. (Insets) Higher magnification of inflammatory infiltrate areas. Original magnification, $400 \times$.
IFN- $\beta$ mRNA, detected by polymerase chain reaction (PCR), was more strongly expressed in L-lep versus T-lep lesions and L-lep versus RR lesions [analysis of variance (ANOVA), $P<0.05$, Fig. 2A]. The mRNA expression for IFNAR1, encoding one of the type I IFN receptors, was more strongly expressed in L-lep versus either T-lep or RR lesions (Fig. 2A). In contrast, IFN- $\gamma$ mRNA was more highly expressed in both T-lep and RR versus L-lep lesions (Kruskal-Wallis, $P<$ 0.05 , Fig. 2A), consistent with previous findings by in situ hybridization (13) and PCR $(4,5)$. IFN- $\beta$ and IFNAR1 protein expression was also more evident in L-lep than T-lep or RR lesions, being present in cells throughout the granuloma (Fig. $2 \mathrm{~B}$ and fig. S8). IFN- $\beta$ protein was found to localize in macrophages in L-lep lesions (figs. S9 to S11) colocalizing with CD14, CD209, and CD163 (14). The elevated expression of IL-10 transcripts in L-lep versus T-lep and RR in lesions, as well as other classic type I IFN-inducible genes, was also corroborated by PCR (Fig. 3A and fig. S12). IL-10 protein was also more highly expressed in L-lep lesions (Fig. 3B), in macrophages as well as T cells (figs. S13 and S14). Double immunofluorescence revealed that IFN- $\beta$ and IL-10 are coexpressed in L-lep lesions, with some cells expressing individual cytokines (Fig. $3 \mathrm{C}$ and fig. S15).

Both live and sonicated M. leprae induced IFN- $\beta$ mRNA and protein in vitro (Fig. 3D). Investigation of the relation between IFN- $\beta$ and IL-10 revealed that IFN- $\beta$ was sufficient to in- duce IL-10 secretion (fig. S16) and that M. leprae induction of IL-10 (Fig. 3E) was partially dependent on type I IFN signaling, blocked by about $40 \%$ by monoclonal antibodies (mAbs) against IFNAR2 (anti-IFNAR2) (Fig. 3F and fig. S17) (9). Together, these data provide evidence for an $M$. leprae $\rightarrow$ IFN- $\beta \rightarrow$ IL-10 pathway in L-lep lesions.

Analysis of the IFN- $\gamma$-induced genes in T-lep lesions revealed several antimicrobial pathways, including activation of the vitamin D-dependent antimicrobial pathway, which leads to the induction of the antimicrobial peptides CAMP (cathelicidin) and DEFB4 (beta-defensin 2), previously shown to be involved in antimicrobial activity in leprosy $(14,15)$ and tuberculosis $(1,11,16,17)$. The gene expression data confirmed the differential expression in T-lep and RR versus L-lep lesions of CYP27B1 mRNA, which encodes the vitamin $\mathrm{D}-1 \alpha$-hydroxylase that converts the prohormone 25-hydroxyvitamin $\mathrm{D}[25(\mathrm{OH}) \mathrm{D}]$ to the bioactive 1,25-dihydroxyvitamin $\mathrm{D}\left[1,25(\mathrm{OH})_{2} \mathrm{D}\right]$ form, as well as the mRNA for the vitamin $\mathrm{D}$ receptor (VDR), the transducer of $1,25(\mathrm{OH})_{2} \mathrm{D}$-directed action in the nucleus of the cell (Fig. 4A). The expression of both CYP27B1 and the VDR were inversely correlated with IL-10 expression in the lesions (fig. S18).

On the basis of the differential expression of type I versus type II IFN-inducible pathways in leprosy, we hypothesized that IFN- $\beta$ and IL-10 inhibited the IFN- $\gamma$-induced antimicrobial pathway. The ability of IFN- $\gamma$ to up-regulate CYP27B1
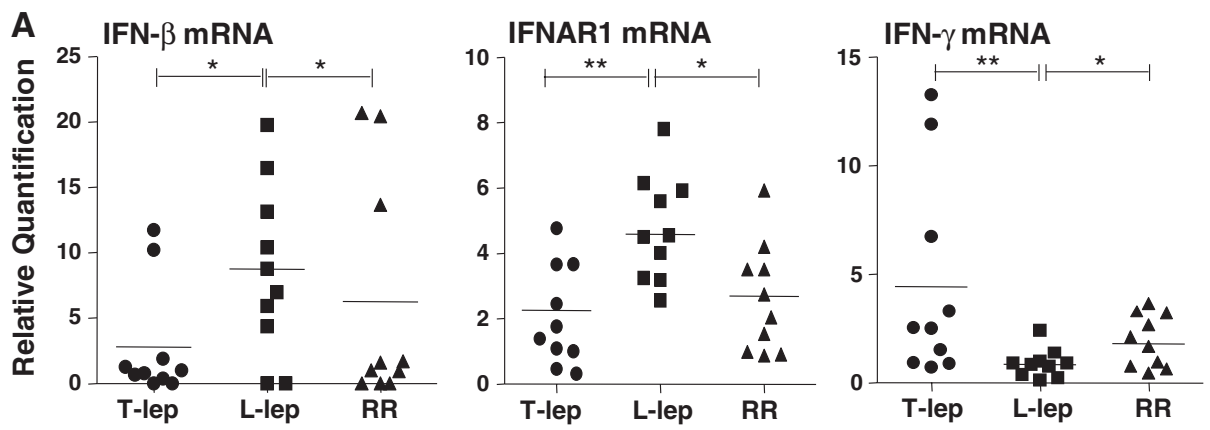

B
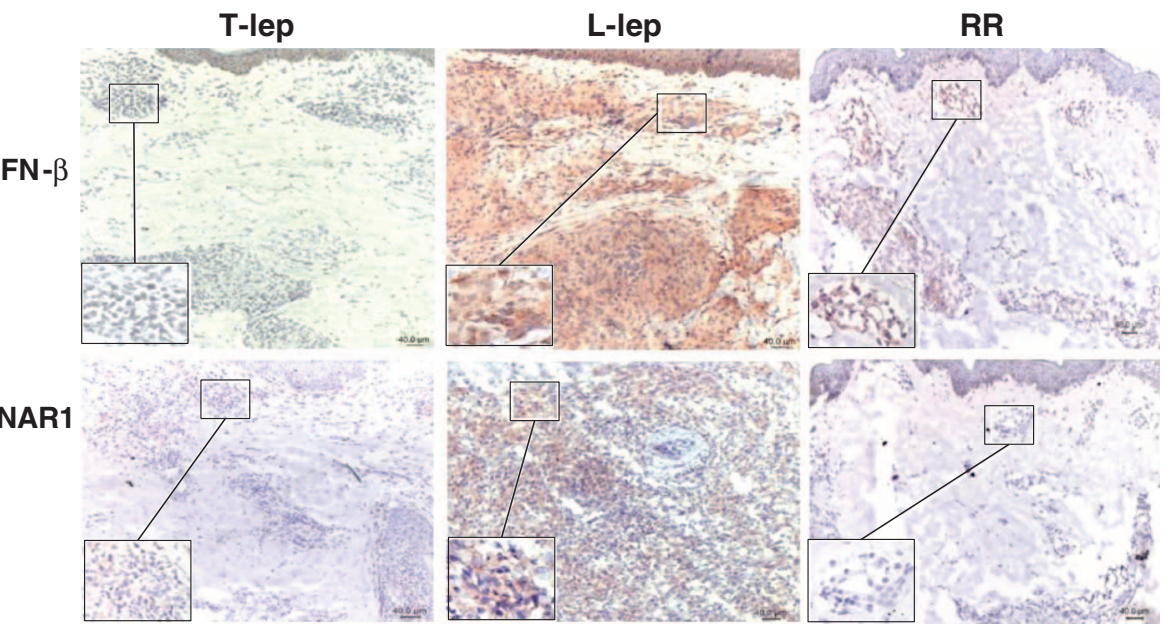
and VDR expression was completely blocked by the addition of IFN- $\beta$ or IL-10 (fig. S19). In addition, the ability of IFN- $\beta$ to inhibit IFN- $\gamma$ induction of CYP27B1 and VDR was reversed by the addition of neutralizing monoclonal anti-IL10 (fig. S19). IL-10 also inhibited IFN- $\gamma$ induction of CYP27B1 activity in macrophages (18), blocking the metabolic conversion of $25(\mathrm{OH}) \mathrm{D}$ to bioactive $1,25(\mathrm{OH})_{2} \mathrm{D}$ (19) (Fig. 4B). Although IFN- $\gamma$ weakly inhibited the vitamin D24-hydroxylase activity [catabolism of $25(\mathrm{OH}) \mathrm{D}$ to the inactive $24,25(\mathrm{OH})_{2} \mathrm{D}$ metabolite], this was also reversed by the addition of IL-10 (fig. S20). It should be noted that the $\mathrm{T}_{\mathrm{H}} 2$ cytokine IL-4, also preferentially expressed in L-lep lesions $(4,20)$, enhances 24 -hydroxylase activity, which inactivates $1,25(\mathrm{OH})_{2} \mathrm{D}(19)$. Therefore, we conclude that IL-10 and IL-4 coordinate to regulate vitamin $\mathrm{D}$ metabolism and catabolism to inhibit IFN- $\boldsymbol{\gamma}$-induced antimicrobial responses.

The ability of IFN- $\gamma$ to up-regulate antimicrobial peptide gene expression, cathelicidin and DEFB4, was completely blocked by addition of IFN- $\beta$ and IL-10 (Fig. 4C) and was reversed by the addition of anti-IL-10 (Fig. 4C). Thus, IFN- $\beta$ was shown to inhibit the expression of key genes involved in antimicrobial activity in human monocytes and macrophages. The effect of type I and II IFNs on the viability of intracellular M. leprae was subsequently investigated in vitro by using an infection model. Because M. leprae cannot be grown in vitro, we measured viability based on the ratio of $M$. leprae $16 S$ rRNA to the $M$. leprae repetitive element DNA $(15,21)$. IFN- $\gamma$ induced an antimicrobial activity against $M$. leprae in monocytes by $\sim 35 \%$, which was blocked $\sim 70 \%$ by pharmacologic inhibition of the VDR (Fig. 4D) and completely abrogated by the addition of either IFN- $\beta$ or IL-10 (Fig. 4F and fig. S21). The ability of IFN- $\beta$ to inhibit the IFN- $\gamma$-induced antimicrobial response against $M$. leprae was almost completely reversed by the addition of anti-IL-10. These studies indicate that the type I IFN program prominently expressed in L-lep lesions inhibits the IFN- $\gamma$-induced antimicrobial response against $M$. leprae, primarily through the intermediation of IL-10 (fig. S22). Additionally, type I IFNs block the ability of inflammasome activators

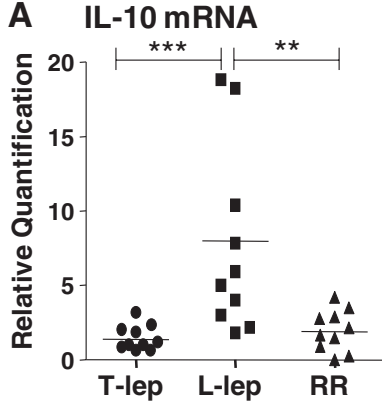

C

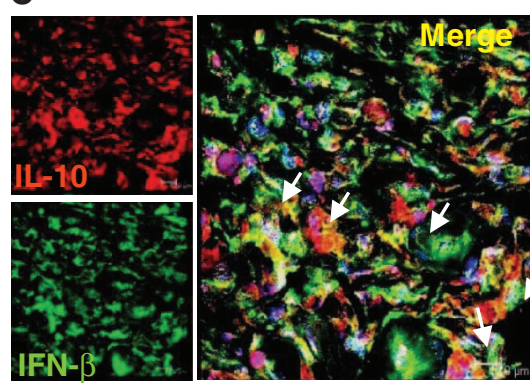

B

IL-10
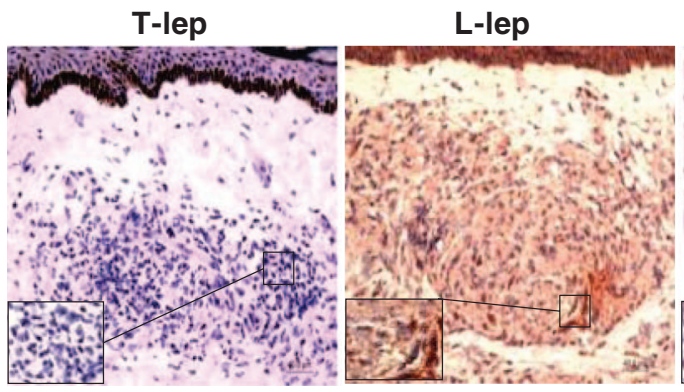

D

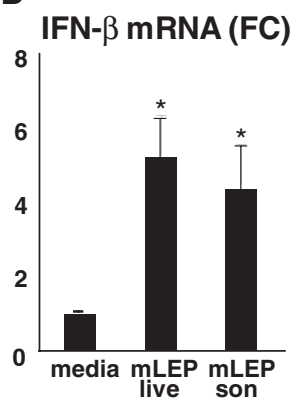

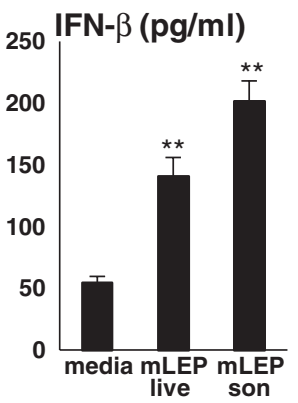
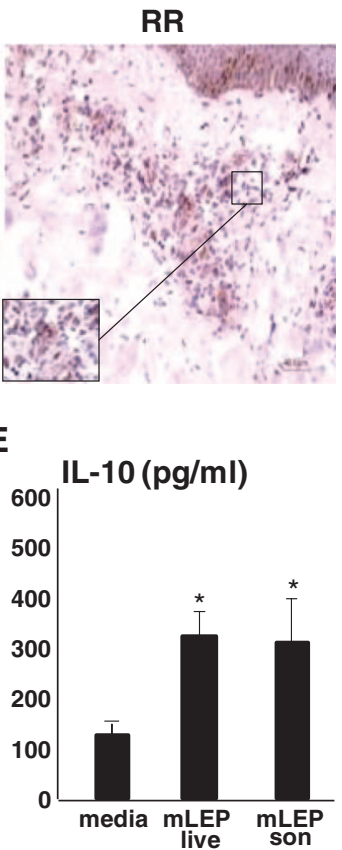

Fig. 3. IL-10 is increased in L-lep lesions and is induced by IFN- $\beta$ and $M$. leprae in vitro. (A) Total mRNA was isolated from L-lep $(n=10)$, T-lep $(n=10)$, and RR $(n=10)$ skin lesions, and IL-10 mRNA levels were analyzed by $\mathrm{qPCR}$. The levels of IL-10 were normalized to GAPDH levels in the same tissue. Statistical significance was calculated by ANOVA followed by Newman-Keuls multiple comparison test. ${ }^{* *} P \leq 0.001 ;{ }^{* * P} \leq$ 0.01. (B) IL-10 expression in leprosy lesions ( $\mathrm{T}$-lep, L-lep, and RR); one representative labeled section is shown out of at least five individuals; scale bars, $40 \mu \mathrm{m}$. Original magnification, 200x. (Insets) Higher magnifi-
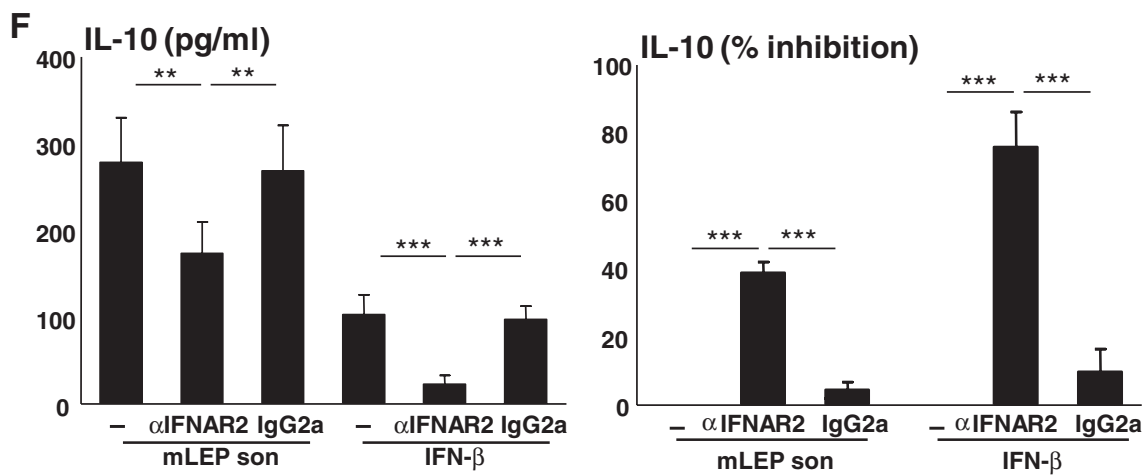

cation of inflammatory infiltrate area. Original magnification, 400×. (C) Colocalization of IFN- $\beta$ (green) and IL-10 (red) in the inflammatory infiltrate of L-lep lesions. Data are representative of three individual L-lep samples; arrows indicate colocalization of the two cytokines. (D) Human monocytes were stimulated with live M. leprae (mLEP) or sonicated mLEP. After 6 hours, qPCR was performed for detection of IFN- $\beta$ (FC, fold change); supernatants were collected after 24 hours for detection of IFN- $\beta$ by enzyme-linked immunosorbent assay. Data are represented as mean \pm SEM, $n=7$. Statistical significance was calculated by two-tailed Student's $t$ test. ${ }^{* *} P \leq 0.01$; ${ }^{*} P \leq 0.05$. (E) Monocytes were stimulated with live mLEP or sonicated mLEP for 24 hours, and IL-10 protein levels were detected. Data are represented as mean \pm SEM, $n=7$. Statistical significance was calculated by two-tailed Student's $t$ test. ${ }^{*} P \leq 0.05$. (F) Human monocytes were stimulated with mLEP sonicated alone or in combination with either human IFNAR2 antibody or isotype control for 24 hours, and IL-10 protein levels were detected. Data are represented as mean $\pm \mathrm{SEM}, n=4$. Left graph shows the levels of IL-10 subtracted from media (average $\pm 64.5 \mathrm{pg} / \mathrm{ml}$ ), and right graph shows the percentage of inhibition of IL-10 levels. Statistical significance was calculated by one-way ANOVA, and comparison between two groups was confirmed by the posttest and Newman-Keuls multiple comparison test. ${ }^{* *} P \leq 0.001 ;{ }^{* *} P \leq 0.01$. IgG2a, immunoglobulin $\gamma 2 \mathrm{a}$. 
(22) or M. tuberculosis infection $(23,24)$ to trigger production of IL-1, a cytokine also required for induction the vitamin D antimicrobial pathway (16). The inverse correlation between the expression of IFN- $\beta$ versus IFN- $\gamma$, as well as their downstream target genes, suggests that the relative expression of IFNs at the site of infection is a key determinant of the outcome of the host response in leprosy. In studying a chronic human disease, it is difficult to separate initial cause from effect, but the data indicate that the IFN- $\beta$ in the fully developed disease is suppressing the development of a protective response. We also note that the relevance of the vitamin D pathway for host defense in leprosy is reflected in the genetic association of VDR single-nucleotide polymorphisms in L-lep patients (25) and the reported successful use of vitamin D as a therapeutic adjuvant in the treatment of leprosy (26).
We believe that these findings in the spectrum of leprosy may provide useful insights into mechanisms of resistance and pathogenesis in the related mycobacterial disease, human tuberculosis. Analogous to leprosy, in tuberculosis, IFN- $\gamma$ is critical for control of the infection. However, in some individuals, the infection with M. tuberculosis progresses to pulmonary and disseminated disease, analogous to the progressive form of leprosy. The most striking characteristic of the blood-based profiling "signature" for active tuberculosis was the increase in a set of genes regulated by $\operatorname{IFN}-\beta(3,12)$, with an overlap in IFN- $\beta$ - and IL-10-induced genes similar to progressive leprosy. The presence of the IL-10 signature profile in blood of tuberculosis patients and the finding of IL-10 mRNA and protein at the site of disease (27) suggest that there is likely to be a causal association between the IFN- $\beta$ and IL-10 profiles in active mycobacterial diseases and tissue damage. This raises the possibility that, in individuals who are able to maintain their M. tuberculosis in a latent or persistent state, a decrease in the type II IFN response or an increase in the type I response, perhaps induced by intercurrent viral infection, could shift the balance from latent to active disease.

We suggest that tuberculosis, like leprosy, comprises a spectrum of protective and pathogenic responses (28). From an evolutionary standpoint, if the immune response were fully effective in killing the pathogen, neither historically ancient disease would exist. When innate and acquired responses are compromised, as in HIV, tuberculosis, often seen as a chronic infectious disease, is transformed into a rapidly fatal disease, as in the case of extensively drug resistant (XDR) tuberculosis (29). Because M. tuberculosis exists
A
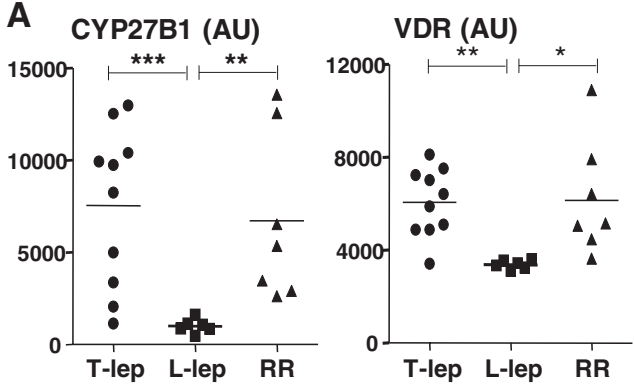

C

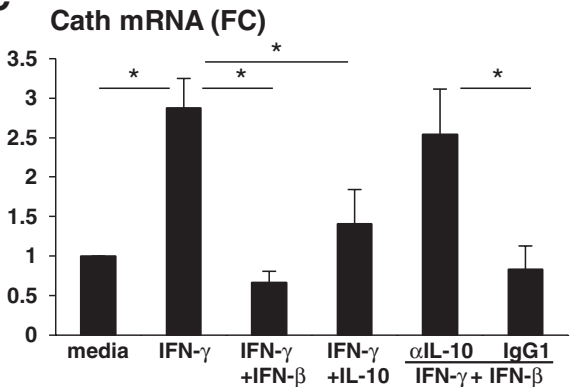

B

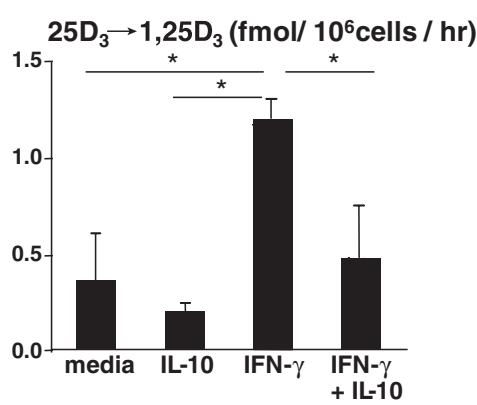

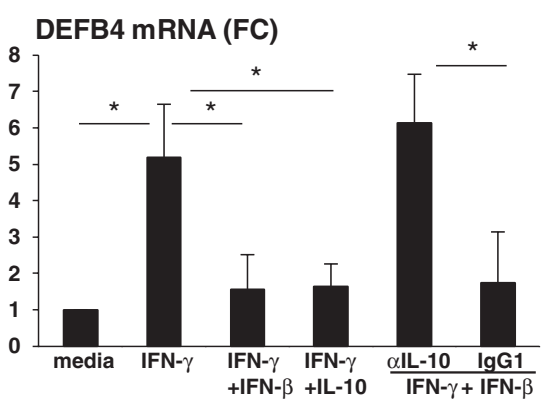

Fig. 4. IFN- $\beta$ and IL-10 inhibit antimicrobial pathway induced by IFN- $\gamma$. (A) Distribution of CYP27B1 and VDR mRNA by microarray analysis of L-lep $(n=6)$, T-lep $(n=10)$, and RR $(n=7)$ skin lesions shown in arbitrary units (AU). Statistical significance was calculated for CYP27B1 by ANOVA followed by Newman-Keuls multiple comparison test and, for VDR, by Kruskal-Wallis followed by Dunn's multiple comparison test. ${ }^{* *} P \leq 0.001 ;{ }^{* *} P \leq 0.01$; ${ }^{*} P \leq 0.05$. (B) Human monocytes were treated with IL-10, IFN- $\gamma$, and IFN- $\gamma$ plus IL-10 for 48 hours followed by incubation with radiolabeled metabolite cholecalciferol (D3), $25(\mathrm{OH}) \mathrm{D}_{3}$ for 5 hours, and the ability to convert $25(\mathrm{OH}) \mathrm{D}_{3}$ to $1,25(\mathrm{OH})_{2} \mathrm{D}_{3}$ was measured by HLPC. Enzymatic conversion data are represented as mean \pm SEM and show three different donors, each studied in triplicate. Statistical significance was calculated by one-way ANOVA repeated measures test followed by Newman-Keuls multiple comparison test. ${ }^{*} P \leq 0.05$. (C) Human monocytes were stimulated with IFN- $\gamma$ alone or in combination with IFN- $\beta$ and IL-10, antibody against human IL-10 was added in the monocyte culture in combination with IFN- $\gamma+$ IFN- $\beta$ for 24 hours, and the cathelicidin (Cath) and DEFB4 mRNA levels were detected by qPCR. Data are represented as mean \pm SEM, $n=7$. Statistical significance was calculated by two-tailed Student's $t$ test. ${ }^{*} P \leq 0.05$. (D)

D

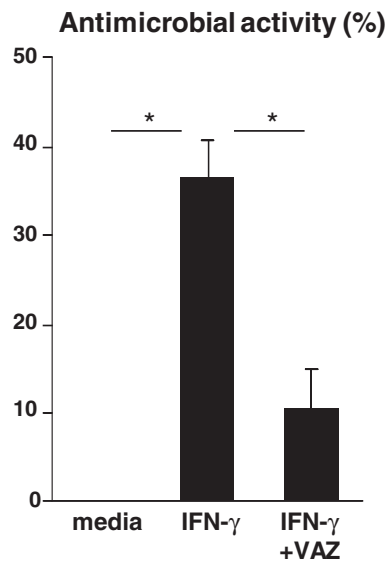

E

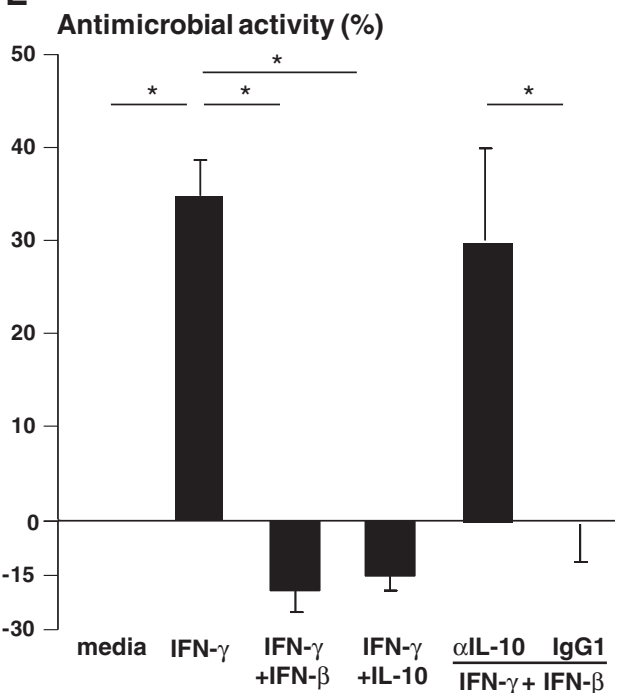
Human monocytes were pretreated with IFN- $\gamma$ and then infected with $M$. leprae at a multiplicity of infection of 10:1 overnight. After infection, cells were treated with IFN- $\gamma$ alone or in combination with VDR antagonist (VAZ). Viability of mLEP was calculated by the ratio of bacterial $16 S$ RNA and DNA (RLEP) detected by qPCR, and percent increase or decrease relative to no treatment (media) was determined. Data are represented as mean \pm SEM, $n=5$. Statistical significance was calculated by two-tailed Student's $t$ test. * $P \leq 0.05$. (E) Human monocytes were pretreated and infected as described in (D). After infection, cells were treated with IFN- $\gamma$ alone or in combination with IFN- $\beta$ or IL-10; in some instances, human IL-10 antibody was added to the culture in combination with IFN- $\gamma$ plus IFN- $\beta$ for 4 days. Viability of $M$. leprae was calculated as described in (D). Data are represented as mean $\pm \mathrm{SEM}, n=10$. Statistical significance was calculated by two-tailed Student's $t$ test. * $P \leq 0.05$. 
essentially only in human species, it is likely to have evolved to persist in the human population, with a sufficient number of individuals developing pathology to assure transmission by aerosol and survival of the pathogen, the remainder containing the pathogen by protective host immune responses. Therapeutic interventions to block IFN$\beta$-induced pathologic responses as well as enhance in IFN- $\gamma$ responses may be an effective strategy to alter the balance to favor protection in mycobacterial and other infections.

\section{References and Notes}

1. M. Fabri et al., Sci. Transl. Med. 3, 104ra102 (2011)

2. R. M. O'Connell et al., J. Exp. Med. 200, 437 (2004).

3. M. P. Berry et al., Nature 466, 973 (2010).

4. M. Yamamura et al., Science 254, 277 (1991).

5. M. Yamamura et al., J. Immunol. 149, 1470 (1992).

6. S. J. Waddell et al., PLOS ONE 5, e9753 (2010).

7. S. A. Samarajiwa, S. Forster, K. Auchettl, P. J. Hertzog, Nucleic Acids Res. 37, D852 (2009).

8. T. R. Golub et al., Science 286, 531 (1999).

9. S. S. Iyer, A. A. Ghaffari, G. Cheng, J. Immunol. 185, 6599 (2010).

10. M. Rayamajhi, J. Humann, K. Penheiter, K. Andreasen, L. L. Lenz, J. Exp. Med. 207, 327 (2010).
11. P. T. Liu et al., Science 311, 1770 (2006); 10.1126/science.1123933.

12. ]. Maertzdorf et al., Proc. Natl. Acad. Sci. U.S.A. 109, 7853 (2012).

13. C. L. Cooper et al., J. Exp. Med. 169, 1565 (1989).

14. D. Montoya et al., Cell Host Microbe 6, 343 (2009).

15. P. T. Liu et al., Nat. Med. 18, 267 (2012).

16. P. T. Liu et al., PLOS ONE 4, e5810 (2009).

17. S. R. Krutzik et al., J. Immunol. 181, 7115 (2008).

18. J. S. Adams, M. A. Gacad, J. Exp. Med. 161, 755 (1985).

19. K. Edfeldt et al., Proc. Natl. Acad. Sci. U.S.A. 107, 22593 (2010)

20. P. Salgame et al., Science 254, 279 (1991).

21. A. N. Martinez et al., J. Clin. Microbiol. 47, 2124 (2009).

22. G. Guarda et al., Immunity 34, 213 (2011).

23. A. Novikov et al., J. Immunol. 187, 2540 (2011).

24. K. D. Mayer-Barber et al., J. Immunol. 184, 3326 (2010)

25. S. Roy et al., J. Infect. Dis. 179, 187 (1999).

26. G. Herrera, Int. J. Lepr. 17, 35 (1949).

27. P. F. Barnes et al., Infect. Immun. 61, 3482 (1993).

28. C. E. Barry 3rd et al., Nat. Rev. Microbiol. 7, 845 (2009).

29. N. R. Gandhi et al., Lancet 368, 1575 (2006).

Acknowledgments: We thank P. Liu for advice with the M. leprae viability assays and helpful discussion; M. So fo

help in the bioinformatic analysis; K. Zavala for help with the high-performance liquid chromatography (HPLC) assays; A. Legaspi and A. DeLeon for help with the immunohistochemistry; M. Schibler and the University of California-Los Angeles, California NanoSystems Institute, Advanced Light Microscopy Core Facility for assistance with the confocal studies; D. Elashoff for help with the statistical analysis; and A. Steinmeyer, E. May, and U. Zügel from Bayer Pharma AG for the VDR antagonist ZK 159222 (VAZ). The live M. leprae was provided by the U.S. National Hansen's Disease Programs through the generous support of the American Leprosy Missions and Society of St. Lazarus of Jerusalem. The data presented in this manuscript are tabulated in the main paper and supplementary materials. Gene expression files containing array data are available under the accession nos. GSE17763 and GSE43700 in the Gene Expression Omnibus (GEO) database. This work was supported in parts by $\mathrm{NIH}$ grants (P50 AR063020; R01s Al022553, AR040312, and Al047868; and Clinical and Translational Science Award grant UL1TR000124)

\section{Supplementary Materials}

www.sciencemag.org/cgi/content/full/science.1233665/DC1 Materials and Methods

Figs. $\mathrm{S} 1$ to $\mathrm{S} 22$

References (30-38)

5 December 2012; accepted 29 January 2013

Published online 28 February 2013;

10.1126/science. 1233665

\section{Dual Origin of the Epithelium of the Mammalian Middle Ear}

\section{Hannah Thompson and Abigail S. Tucker*}

The air-filled cavity and ossicles of the mammalian middle ear conduct sound to the cochlea. Using transgenic mice, we show that the mammalian middle ear develops through cavitation of a neural crest mass. These cells, which previously underwent an epithelial-to-mesenchymal transformation upon leaving the neural tube, undergo a mesenchymal-to-epithelial transformation to form a lining continuous with the endodermally derived auditory tube. The epithelium derived from endodermal cells, which surrounds the auditory tube and eardrum, develops cilia, whereas the neural crest-derived epithelium does not. Thus, the cilia critical to clearing pathogenic infections from the middle ear are distributed according to developmental derivations. A different process of cavitation appears evident in birds and reptiles, indicating that this dual epithelium may be unique to mammals.

$\mathrm{T}$ he mammalian middle ear is an air-filled cavity housed within the auditory bulla with three ossicles suspended within it, connecting the eardrum to the inner ear. The epithelial lining of the middle ear in the ventral region is continuous with the auditory (Eustachian) tube, which connects the middle ear to the pharynx. At embryonic day 12.5 (E12.5) in the mouse, the ossicles condense within the neural crestderived first and second pharyngeal arches, adjacent to the developing inner ear and dorsal to the tip of the first pharyngeal pouch (1). In early postnatal mice, the future middle ear cavity is filled with neural crest cells surrounding the developing ossicles (2), which are positioned in the dorsal region of the future cavity (the attic), in addition to mesodermal cells that will mature to form the middle ear muscles. A process called

Department of Craniofacial Development and Stem Cell Biology, King's College London, Guy's Hospital, London, UK, SE1 9RT. *Corresponding author: abigail.tucker@kcl.ac.uk cavitation then occurs in which the neural crest cells are replaced by an air-filled cavity $(2,3)$, which surrounds the ossicles and muscles, allowing free movement in response to sound (fig. S1). The whole cavity is lined by an epithelium.

The current model of middle ear cavitation was proposed by Wittmaack (4) and suggests that the endoderm of the first pharyngeal pouch extends into the middle ear region, expanding and enveloping the middle ear structures, resulting in a cavity lined completely by endoderm. However, the middle ear cavity has suspended within it three ossicles in addition to muscles, blood vessels, and nerves that would prevent an epithelium expanding through as a continuous sheet. This prompted Schwarzbart to propose that during cavitation the endoderm ruptured and the neural crest formed the lining of the middle ear (5). To resolve these issues, we have made use of newly developed transgenic mouse lines Sox 172Aicre (6) and Wntlcre (7) crossed with the reporter mouse line $R 26 R(8)$. When stained with $\mathrm{X}-\mathrm{Gal}$, this system permanently labels cells of endodermal or neural crest origin blue and therefore allows the embryonic origin of tissues within the developing middle ear to be determined.

Sox 17-2AicreR26R mice trace cells that are currently expressing, or have previously expressed, Sox17. These include cells of endodermal origin and blood vessels. Contrary to previously published data that the epithelial lining of the middle ear is of endodermal origin $(9,10)$, the fully cavitated middle ear from P14 in Sox17-2AicreR26R mice was found to be labeled blue only in the epithelium around the opening to the auditory tube (Fig. 1B), with unstained epithelium around the attic region above the ossicles and along the cochlea (Fig. 1A). To determine the origin of the nonendodermal epithelial cells, Wnt1creR26R mice were stained with X-Gal. Around the auditory tube, the epithelium was LacZ-negative (Fig. 1D), whereas the epithelium lining the cavity around the ossicles and cochlea was LacZ-positive (Fig. 1C), in a complementary pattern to that observed in the Sox17-cre line. Careful mapping of the middle ear cavity with these reporter lines showed that the auditory tube and surrounding middle ear epithelium, extending up to and slightly beyond the eardrum on the lateral side, was endoderm-derived. In contrast, neural crest cells were found lining the middle ear cavity on the medial wall covering the otic capsule/cochlea and lining the attic region of the cavity in the vicinity of the ossicles (Fig. 1E) (number of samples analyzed $=15$ ). In humans, the attic is connected to the mastoid air space, and these are therefore also likely to be lined with a neural crestderived epithelium. The epithelium lining the middle ear cavity is therefore of dual origin, roughly half neural crest and half endodermal. 
If you wish to distribute this article to others, you can order high-quality copies for your colleagues, clients, or customers by clicking here.

Permission to republish or repurpose articles or portions of articles can be obtained by following the guidelines here.

The following resources related to this article are available online at www.sciencemag.org (this information is current as of March 9, 2015 ):

Updated information and services, including high-resolution figures, can be found in the online version of this article at:

http://www.sciencemag.org/content/339/6126/1448.full.html

Supporting Online Material can be found at:

http://www.sciencemag.org/content/suppl/2013/02/27/science.1233665.DC1.html

A list of selected additional articles on the Science Web sites related to this article can be found at:

http://www.sciencemag.org/content/339/6126/1448.full.html\#related

This article cites 38 articles, 26 of which can be accessed free:

http://www.sciencemag.org/content/339/6126/1448.full.html\#ref-list-1

This article has been cited by 24 articles hosted by HighWire Press; see:

http://www.sciencemag.org/content/339/6126/1448.full.html\#related-urls

This article appears in the following subject collections:

Immunology

http://www.sciencemag.org/cgi/collection/immunology 